\title{
Highly-Accurate Real-Time Syntonization Using A GPS Single-Frequency Receiver With An Intelligent Atmosphere Forecasting Model
}

\author{
C.-L. CHENG ${ }^{1}$, F.-R. CHANG ${ }^{1}$, L.-S. WANG ${ }^{2}$, K.-Y. TU ${ }^{3}$ \\ 1. Dept. of Electrical Engineering, National Taiwan University. \\ 2. Inst. of Applied Mechanics, National Taiwan University. \\ 3. NSTF Lab., TL, Chunghwa Telecom. Co., Ltd., Taiwan. \\ National Taiwan University, Dept. of Electrical ENG., RM207, Taipei 106, Taiwan. \\ Tel: +886-2-23635251 ext.207, Fax: +886-2-23638247, Email: randy7@ms36.hinet.net
}

\begin{abstract}
A low-cost, highly-accurate real-time GPS carrier phase frequency syntonization system based on a single-frequency receiver is presented. By using the atmosphere free model of the carrier phase with the neural-wavelet filter, and performing the time-difference operation, the low-cost oscillator can be automatically steered to obtain very high frequency accuracy and stability in the short term ( 1 sec.) as well as in the long term (1 day).
\end{abstract}

\section{INTRODUCTION}

The frequency source (FS) plays a key role in many applications, such as telecommunication networks, power systems, navigation systems, instrumentation systems, and Doppler radars, etc. The Global Positioning System Disciplined Oscillator (GPSDO), based on C/A code observations, is one of the principal methods of maintaining highly accurate frequency dissemination worldwide. However, GPSDOs are subject to errors or biases caused by signal noise and the atmosphere. The possibility of using the GPS carrier phase, rather than C/A code, for the precise time and frequency transfer has been described by many researchers [1]. Because the frequency of the carrier phase is roughly 1000 times higher than that of $\mathrm{C} / \mathrm{A}$ code, time and frequency dissemination using the carrier phase should provide much greater resolution, in principle. To achieve the highest frequency accuracies from the GPS system, the atmospheric propagation errors should be neutralized and considered within the adjustment process. Currently, on the one hand, atmospheric errors may be minimized by a multi-frequency receiver with or without the common view method, but this type of receiver is either more expensive or less reliable than a single-frequency receiver. Therefore, we here introduce a less communication-dependent, low-cost, yet accurate syntonization system composed of a single-frequency GPS carrier phase receiver with an intelligent atmosphere-forecasting model. On the other hand, single-frequency receivers usually include a correction for the atmosphere delay based on an ionosphere model and a troposphere model built into the GPS system. These models are expected to eliminate about $40 \% \sim 75 \%$ of the atmospheric effects on average. Since the parameters of these models are estimated in advance and then transmitted to the GPS satellites, they cannot anticipate day-to-day random fluctuations and thus cannot be completely accurate in real time. Alternatively, various organizations have developed detailed and accurate models of the atmosphere based on GPS observations for single-frequency users to reduce atmospheric effects as much as possible in post-processing. However, the accuracy of these models may vary according to computational complexity and user location. To compensate for the above drawbacks, we have developed an accurate real-time atmosphere forecasting model composed of a neural-wavelet technique. A low-cost GPS receiver, Ashtech G12, was modified in order to estimate the real-time average frequency deviation of the steered Oven-Controlled Crystal Oscillator (OCXO) with respect to the GPS system time by performing the time-difference, all-satellites-in-view average, and atmospheric correction. The scheme can achieve traceability in frequency dissemination. The non-decimated wavelet method, neural networks, and neural network MPC (Model Predictive Controller) were employed to implement the syntonization system. The steered clock was then syntonized with the GPS system time by way of the D/A converters.

With the above methods, the steered clock (OCXO) showed that the accuracy could be improved from about two parts in $10^{9}$ to about three parts in $10^{14}$, and the stability of the syntonized clock could be improved from about nine parts in $10^{10}$ to about four parts in $10^{14}$ for an averaging time of one day. Our experiments revealed that the proposed architecture is sound and cost-effective.

This paper is organized as follows:

1) Section 2 introduces the model of the GPS carrier phase observations.

2) Section 3 presents the intelligent atmosphere forecasting 
model with a neural-wavelet network.

3) Section 4 describes the system architecture.

4) The experimental results are illustrated in Section 5 .

5) Finally, the concluding remarks are given in Section 6.

\section{THE MODEL OF GPS CARRIER PHASE OBSERVABLES}

The typical model of GPS carrier phase observables [2] is shown in equation (1):

$$
\Phi_{A}^{j}=\rho_{A}^{j}+c\left(d t^{j}-d T_{A}\right)+\lambda N_{A}^{j}-d_{i o n}^{j}+d_{\text {trop }}^{j}+\varepsilon_{A}^{j}
$$

where

$\Phi_{A}^{j}$ : Carrier phase measurement of the receiver A from the j-th GPS satellite;

$\rho_{A}^{j}$ : True distance between the receiver A and the $\mathrm{j}$-th GPS satellite;

c: Speed of light;

$d t^{j}$ : Clock bias of the $\mathrm{j}$-th satellite;

$d T_{A}:$ Clock difference between the GPS time and receiver A clock;

$\lambda$ : GPS carrier phase wavelength;

$N_{A}^{j}$ : Initial phase integer ambiguity;

$d_{\text {ion }}^{j}:$ Ionospheric delay;

$d_{\text {trop }}^{j}:$ Tropospheric delay;

$\varepsilon_{A}^{j}$ : Unmodeled errors primarily due to multi-path, temperature variation, physical factors, etc.

The unit of the phase observable $\Phi_{A}^{j}$ in the equation is the meter.

To study the frequency syntonization, we would like to first examine the behavior of the oscillator. Hence, the GPS receiver's internal clock will be replaced by an external one. Under this arrangement, the term $d T_{A}$ in equation (1) represents the time difference between the GPS clock and the external clock A. In our system, the A (user) site performs the time-difference operation (differences between two epochs) on the carrier phase observations to obtain the phase difference with respect to the GPS system time. If the satellite signal is continuously tracked and no cycle slip occurs, the cycle ambiguities $N_{A}^{j}$ remain a constant. After the time difference operation, equation (1) is then rewritten as equation (2):

$$
\delta \Phi_{A}^{j}=\delta \rho_{A}^{j}+c \delta d t^{j}-c \delta d T_{A}-\delta d_{\text {ion }}^{j}+\delta d_{\text {trop }}^{j}+\delta \varepsilon_{A}^{j}
$$

where $\delta(\cdot)$ denotes the operator for the differences between two epochs. The $\delta d T_{A}$ represents the phase difference with respect to the GPS system time. The unmodeled ionospheric delay $\delta d_{i o n}^{j}$ and tropospheric delay $\delta d_{\text {trop }}^{j}$ cannot be eliminated, and they are regarded as errors of the frequency offset.

The B (host) site, stratum 1, installs the same GPS receiver and performs the time difference operation that is the same as that of the A (user) site. Additionally, the A (user) site performs the single difference operation between two receivers at post processing. The two receivers are denoted by $\mathrm{A}$ and $\mathrm{B}$, respectively. The satellite is denoted by $\mathrm{j}$. After the time-difference and single-difference operations, equation (2) is further adapted for equation (3):

$$
\Delta \delta \Phi_{A B}^{j}=\Delta \delta \rho_{A B}^{j}-c \Delta \delta d T_{A B}+\Delta \delta \varepsilon_{A B}^{j}
$$

where $\Delta(\cdot)$ represents the operator for differences between receivers with the same satellite. Due to the strong correlation between the un-modeled ionospheric and tropospheric delays of the two receivers in the local area, the terms $d_{i o n}^{j}$ and $d_{\text {trop }}^{j}$ in equation (1) are then eliminated. Since $d T_{A}$ and $d T_{B}$ are both referring to the same GPS time, their difference $\Delta \delta d T_{A B}$ in (3) is the phase difference between external clock A and external clock B of stratum 1 .

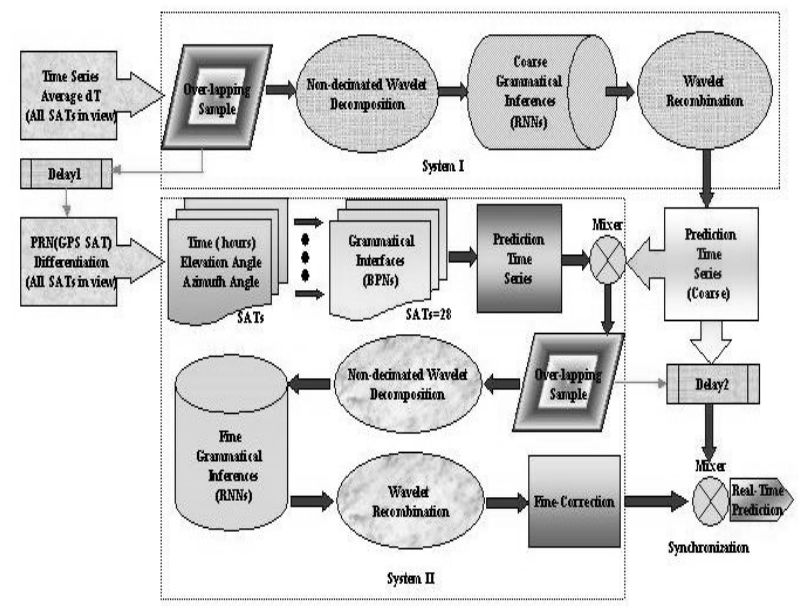

Figure 1. The functional block diagram of the intelligent forecasting model for the average atmosphere delay.

\section{INTELLIGENT ATMOSPHERE FORECASTING MODEL}

Atmospheric forecasting is an example of a signal processing problem which is challenging due to small sizes, high noise, non-stationarity, and non-linearity. We present a novel forecasting strategy that operates at multiple resolutions, using a wavelet transform. The latter exposes useful information, which is then processed by the neural networks. Neural networks provide a valuable framework for the representation of relations present in data. Especially in the fields of classification and time series prediction, neural networks have made substantial contributions. 
The intelligent forecasting model has two main systems. This is illustrated in Figure 1.

System I performs a global approximation of the desired prediction with the real-time limited information composed of high noise, non-stationarity, and non-linearity. Wavelet transforms provide a sensible decomposition of a single or time series, so that faint temporal structures can be revealed and handled by nonparametric models. Two processing strategies are addressed for System I. First, all the wavelet coefficients for a particular time-point are taken together and fed into the input of a Dynamical Recurrent Neural Network (DRNN). These neural network models, endowed with a temporal dimension through embedding autoregressive filters in a fully recurrent neural structure, have powerful representation capabilities. Second, the prediction task is subdivided: a DRNN model is run at each resolution level to provide corresponding estimates. The latter are then combined to form the forecast for the average atmosphere delay series. This allows not only for a modular approach but also for what could be informally described as a mixture of experts: other forecasting techniques may be employed conjointly and modeled at different levels of resolution. The correction of System II is available for fine-tuning the coarse prediction of System I by performing a local approximation. Two main networks are included. The first network uses three parameters, time, elevation angle, and azimuth angle, to create an average atmosphere forecasting model. Other multi-layer perception neural networks, which feed-forward with error back-propagation, are applied to model the past atmosphere average delays for the 28 GPS satellites [3]. This model allows for less interference with the input data when it is modeled by a neural network. The second network delivers the difference signal between System I and the parametric model of System II to another neural-wavelet network trained to estimate the fine-tuning calibration for System I.

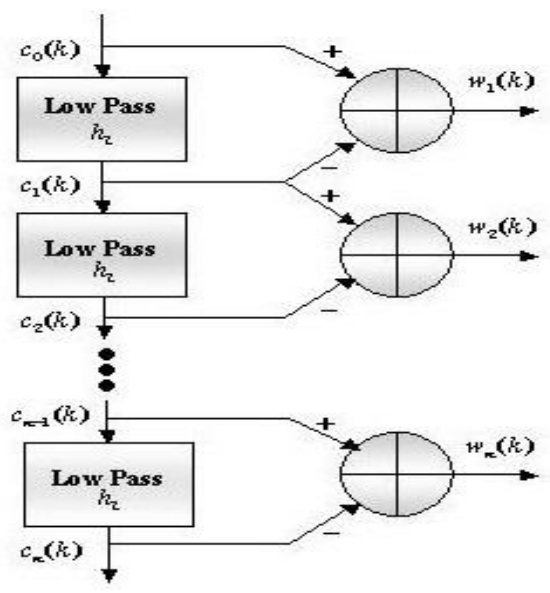

Figure 2. Non-decimated wavelet transform of a time-series signal.

\section{A. The Non-decimated Wavelet Decomposition}

Overlapping window sample methods and non-decimated wavelet techniques are implemented in our proposed model. The overlapping window sample is pre-proceeded with the wavelet technique for use in real time. In addition, the overlapping sample must be adopted into the neural learning target data to avoid aliasing in wavelet recombination. The window length and shift of each sample should be traded off to obtain enough signal information on wavelet decomposition and to avoid a long setup time.

The non-decimated wavelet transform (NWT) overcomes the problem encountered with discrete wavelet transform (DWT) by using a redundant basis in which it produces equal-length wavelet coefficients for each resolution level. Redundancy is helpful in detecting fine features of the detail signals since no aliasing biases arise through decimation. The algorithm of the NWT is the same as that of the DWT except that there is no decimation step [4]. If we consider a given time series signal $c_{0}(k)$, the DWT is performed by passing the signal through a series of low pass filters, $h_{l}$. The result obtained at the output of each filter is the approximation (low frequency information) coefficient series. The number of times the signal is filtered depends on the highest resolution level determined for the filtering process. That is, if the highest resolution level set is $n$, the signal will be filtered $\mathrm{n}$ times with a chain of approximation coefficient series $c_{n}(k)$ obtained at each of the different resolution levels. The expression that describes this process is given as

$$
c_{j}(k)=\sum_{l=0}^{L-1} h_{l} c_{j-1}\left(k+2^{j-1} l\right)
$$

In addition to producing an approximation coefficient series at level j, the NWT wavelet transform also generates the wavelet (high frequency information) coefficient series. Referring to equation (5), the wavelet coefficient series is obtained by taking the difference between $c_{j-1}(k)$ and $c_{j}(k)$.

$$
\mathrm{w}_{\mathrm{j}}(k)=c_{j-1}(\mathrm{k})-\mathrm{c}_{\mathrm{j}}(\mathrm{k})
$$

The generation of the wavelet coefficient series is further illustrated with the block diagram shown in Figure 2.

Last, the signal can be reconstructed using the mathematical expression described in equation (6).

$$
c_{0}(k)=c_{n}+\sum_{j=1}^{n} w_{j}(k)
$$

\section{B. The DRNN Model}

The use of a dynamic recurrent neural network is also 
important for our system in consideration of the curse of dimensionality because the RNN can take into account the greater history of the input [5]. The Elman recurrent neural network was chosen because it is suitable for the grammatical inference style problem, and because it has been shown to perform well in comparison to other recurrent architectures.

The raw time series values of phase error function are $\Phi(k), k=1,2, \ldots, N$ where $\Phi(k)$, phase error function $\in \Re$. These denote the phase error time series of the receiver external clock. The first difference for the time series of phase error function, $\delta \Phi(k)$, is taken as follows:

$$
\delta \Phi(k)=\Phi(k)-\Phi(k-1)
$$

This produces $\delta \Phi(k), \delta \Phi(k) \in \mathfrak{R}, k=1,2, \ldots, N-1$. In order to compress the dynamic range of the series and reduce the effects of the outliers, a log transformation of data is used:

$$
m(k)=\operatorname{sign}(\delta \Phi(k))(\log (|\delta \Phi(k)|+1))
$$

resulting in $m(k), k=1,2, \ldots, N-1, m(k) \in \mathfrak{R}$. As is usual, a delay embedding of this series is then considered:

$$
\mathbf{M}\left(k ; d_{1}\right)=\left(m(k), m(k-1), m(k-2), \ldots, m\left(k-d_{1}+1\right)\right.
$$

where $d_{1}$ is the delay embedding dimension and equal to 1 for the experiments reported here. $\mathbf{M}\left(k ; d_{1}\right)$ is a state vector. This delay embedding forms the input to the scaling function. Hence, the scaling function input is a vector of the last $d_{1}$ values of $\log$ transformed differenced time series. The output of the scaling function always falls within a specified range. Then, the neural network training can be made more efficient for the network inputs and targets.

The scaling function can be represented by the following equation (10):

$$
S(k)=g(\mathbf{M}(k, d))
$$

where $S(k) \in\left[1,2,3, \ldots, n_{s}\right]$, and $n_{s}$ is the number of embedded time series for the scaling function. If the scaling function is used to preprocess the training set data, then each time the trained network is used with new inputs, they should be preprocessed with the same parameters of scaling that were computed for the training set.

For the Elman network [6], [7]:

and

$$
\mathbf{O}(k+1)=\mathbf{C}^{\mathbf{T}} \mathbf{z}_{\mathbf{k}}+\mathbf{C}_{\mathbf{0}}
$$

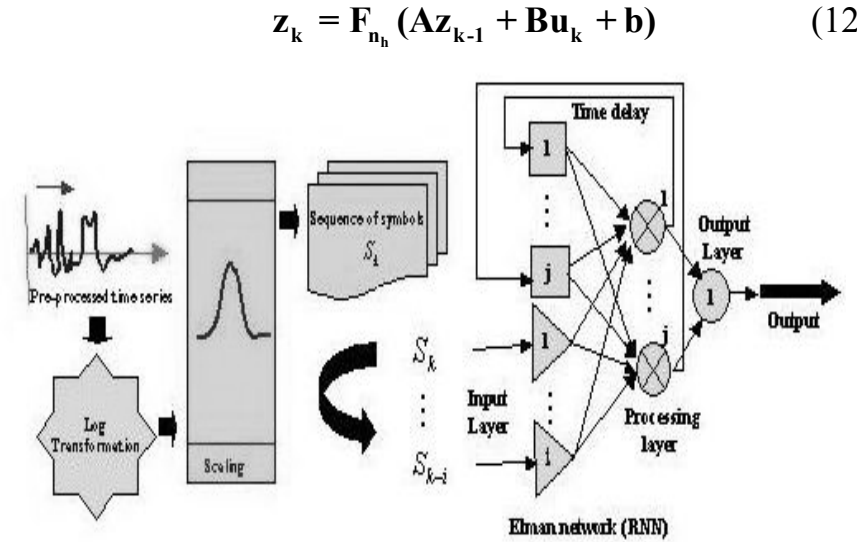

Figure 3.: An DRNN is trained on a sequence of scaling time series (The pre-processed, delay embedded series is converted into a specified range using a scaling function).

where $\mathbf{C}$ is an $n_{h} \times n_{0}$ vector representing the weights from the hidden (processing) layer to the output nodes, $n_{h}$ is the number of the hidden nodes, $n_{0}$ is the number of the output nodes, $\mathbf{C}_{\mathbf{0}}$ is a constant bias vector, $\mathbf{z}_{\mathbf{K}}, \in \mathfrak{R}^{n_{h}}$, and is an $n_{h} \times 1$ vector, denoting the outputs of the hidden layer neurons. $\mathbf{u}_{\mathbf{k}}$ is a $d_{2} \times 1$ vector as follows, where $d_{2}$ is the embedding dimension used for the recurrent neural network.

$$
\mathbf{u}_{\mathbf{k}}=\left[\begin{array}{c}
S(k) \\
S(k-1) \\
\vdots \\
S\left(k-d_{2}+1\right)
\end{array}\right]
$$

A and $\mathbf{B}$ are matrices of appropriate dimensions which represent the feedback weights from the hidden layer to the hidden notes, and the weights from the input layer to the hidden layer, respectively. $F_{n_{h}}$ is an $n_{h} \times 1$ vector containing the sigmoid functions. One benefit of a sigmoid function is that it reduces the effect of extreme input values, thus providing some degree of robustness to the network. The $\mathbf{b}$ is an $n_{h} \times 1$ vector, denoting the bias of each hidden layer neuron. $\mathbf{O}(k)$ is an $n_{0} \times 1$ vector containing the outputs of the network. $n_{0}$ is 1 throughout this paper. Thus, for the completed system:

$$
\mathbf{O}(k+1)=F_{1}(\delta \Phi(k), \delta \Phi(k-1), \ldots, \delta \Phi(k-4))
$$

which can be considered in terms of the original time series: 


$$
\mathbf{O}(k+1)=F_{2}(\Phi(k), \Phi(k-1), \ldots, \Phi(k-5))
$$

Note that this is not a static mapping, due to the dependence on the hidden state of the recurrent neural network. However, the forms of the nonlinear function $F_{1}, F_{2}$ are not apparent from the neural network.

\section{The Ability of the Intelligent Forecasting Model}

To verify the ability of our real-time forecasting model, the data from an Ashtech Z-XIIT is preliminarily processed. Now, the ION-free effect of the dual frequency method is the training goal for our forecasting model. We use the information on the past three days to train our model. Then, we simultaneously adopt the dual frequency method and our model to correct the phase difference data between the primary clock and GPS system time for 7 days.

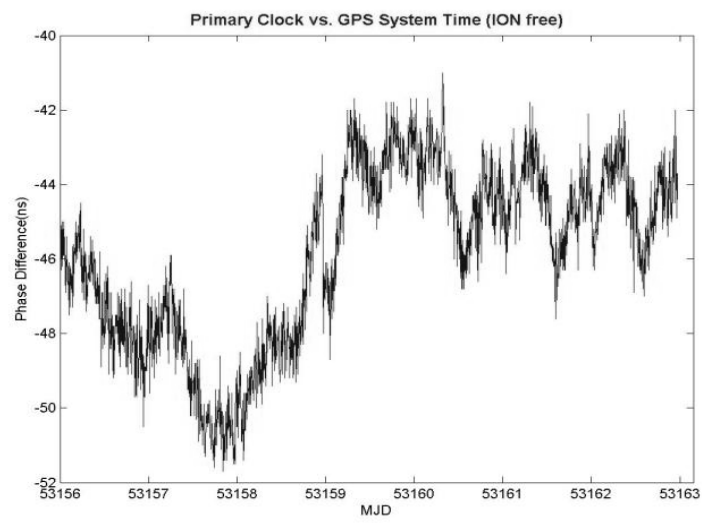

Figure 4. The phase difference estimation between primary clock and GPS system time using the dual-frequency method for ION-delay free.

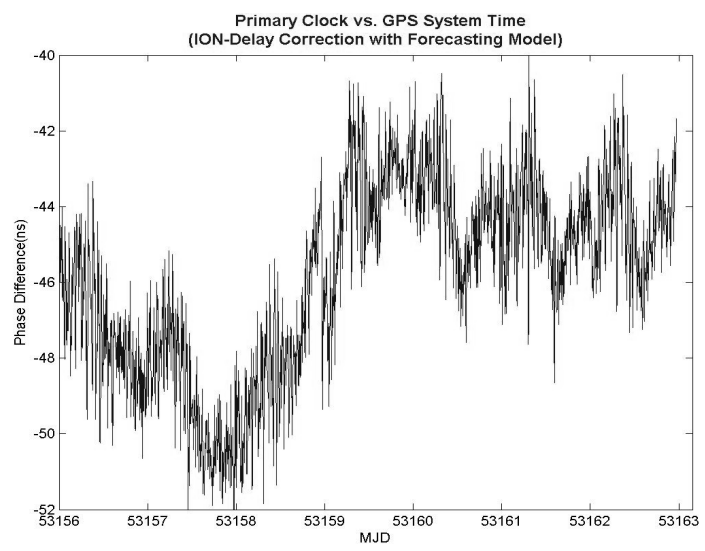

Figure 5. The phase difference estimation between primary clock and GPS system time using the forecasting model for the real-time correction of ION-delay.

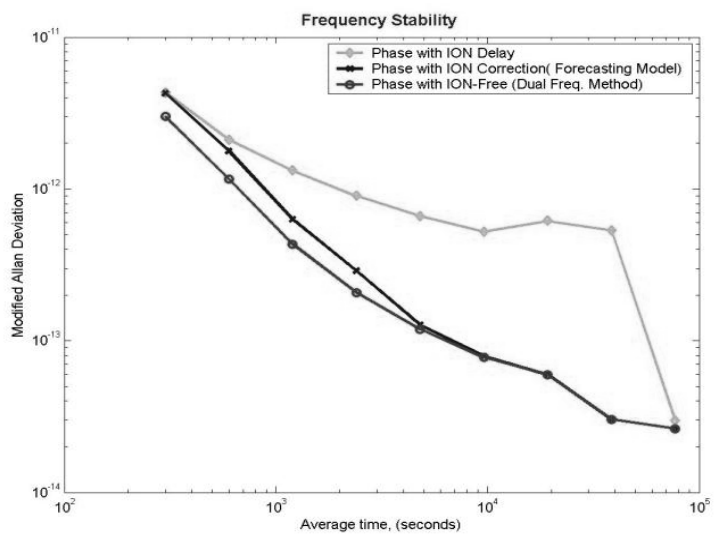

Figure 6. The frequency stability comparison using the two ways of the ION-delay correction, without ION-delay correction

The phase difference estimations produced using these two methods are shown separately, in Figure 4 and Figure 5. Figure 6 expresses the frequency stability analysis. The results indicate that our real-time forecasting model is effective and reliable. The model has the ability to perform continuously with rational accuracy for 7 days. In addition, the model establishment is not relevant to user location, so the accuracy will not vary with latitude. Next, we introduce the forecasting model into the low-cost disciplined oscillator to correct for the atmosphere in real time.

\section{THE ARCHITECTURE OF THE SyNTONIZATION SySTEM}

\section{A. Low-Cost Syntonization System}

The functional block diagram of our syntonization system is shown in Figure 7. This system consists of mainly the DDS (Direct Digital Synthesizer), a D/A converter, a low-cost modified G12 GPS receiver, a forecasting model of atmosphere correction, and a notebook PC. In order to estimate the offsets of the steered clock with respect to the GPS system time, the user clock is connected to the modified GPS receiver. Hence, the original internal quartz oscillator in the receiver is replaced. With the help of the frequency synthesizer, i.e. the DDS (Direct Digital Synthesizer) manufactured by NOVATECH, the signal of the external clock can be appropriately converted and supplied to the GPS receiver. The behavior of the clock then comes into view from the GPS observations. The average frequency offset of the syntonized clock with respect to the GPS system time can be estimated by performing the time difference operation (i.e., (2)) on carrier phase observations. Equation (2) can be further expressed as equation (16):

$$
\delta \Phi_{A}^{j}-\delta \rho_{A}^{j}-c \delta d t^{j}+\delta d_{\text {ion }}^{j}-\delta d_{\text {trop }}^{j}=-c \delta d T_{A}+\delta \varepsilon_{A}^{j}
$$


The left-hand side of the equation (16) is the time difference data of the known measurement. The coordinates of the GPS antenna are predetermined by the IGS (International GPS Service), and the coordinates and the satellite clock error of the $\mathrm{j}$-th GPS satellites are obtained from the broadcast navigation message. Furthermore, the ionospheric delay $\left(\delta d_{i o n}^{j}\right)$ and the tropospheric delay $\left(\delta d_{\text {trop }}^{j}\right)$ are thus eliminated from the real-time average atmospheric delay forecasting model. Some noise errors affecting the estimation of the average frequency offset may occur in the evaluation of the right-hand side.

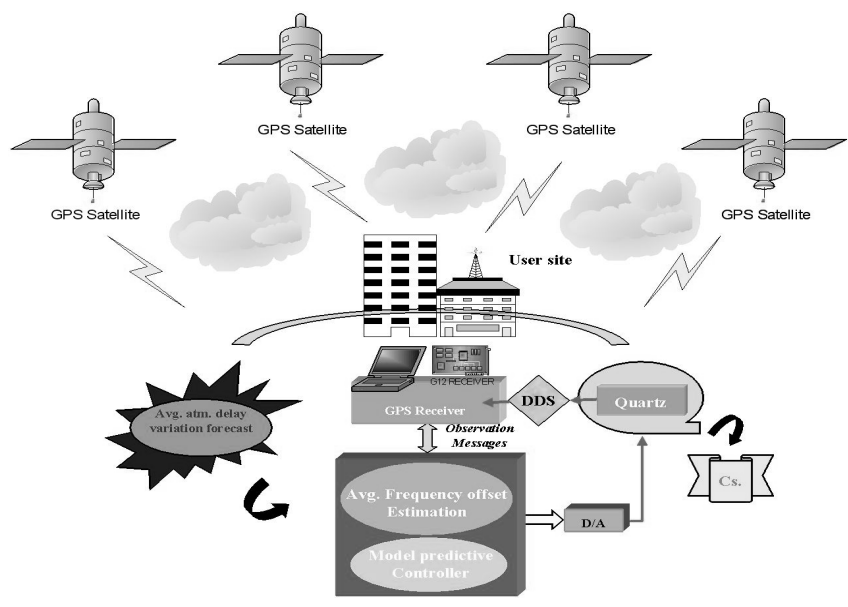

Figure 7 . The functional block diagram of our syntonization system

The term $\delta d T_{A}\left(t_{i}\right)\left(=d T_{A}\left(t_{i}\right)-d T_{A}\left(t_{i-1}\right)\right.$, where $\left.t_{i}=t_{i-1}+\tau\right)$ can be obtained by averaging (16) for all in view GPS observations. As previously mentioned, since $\delta d T_{A}\left(t_{i}\right)$ is the phase difference between the GPS system time, the associated estimate frequency offset $\hat{y}_{r}^{\prime}\left(t_{i}\right)$ is shown in equation (17):

$$
\hat{y}_{r}^{\prime}\left(t_{i}\right)=\frac{\delta d T_{A}\left(t_{i}\right)}{\tau}-\frac{\delta \varepsilon_{A}^{j}}{c \tau}
$$

In general, fine frequency tuning can be performed on an inexpensive oscillator through voltage control. Because of environmental effects such as vibration, temperature, pressure, and humidity, the desired frequency output is not always under a constant voltage.

In our system, the frequency offset $\hat{y}_{r}\left(t_{i}\right)$ is chosen as the input variable of the model predictive controller. An incremental voltage $\Delta v\left(t_{i}\right)$ will be generated to update the voltage to allow steering of the oscillator.

$$
v_{i+1}=v_{i}+\Delta v
$$

For a low-cost quartz or rubidium oscillator, the frequency offset may vary over very large scales. The log transformation of the data is used to compress the values of the estimation $y_{\tau}\left(t_{i}\right)$. The compression function is shown in equation (19):

$$
w\left(t_{i}\right)=\operatorname{sign}\left[y_{\tau}\left(t_{i}\right)\right] \times\left\{a \times \log \left[\left|y_{\tau}\left(t_{i}\right)\right|+b\right]\right\}
$$

where $a$ and $b$ are the scaling factor and the offset factor, respectively. We decide to adopt the model predictive controller (MPC) to steer the external clock because the MPC can systematically take into account real plant constraints in real time. It is also robust with respect to modeling errors, over and under parameterization, and sensor noise [8].

The neural network predictive controller that is implemented in the highly-accurate real-time sytonization uses a neural network model of a nonlinear plant to predict future plant performance. The controller then calculates the control input that will optimize plant $(\mathrm{OCXO})$ performance over a specified future time horizon. The first step in MPC is to determine the neural network plant model. Next, the plant model is used by the controller to predict future performance. The following section will describe the system identification process.

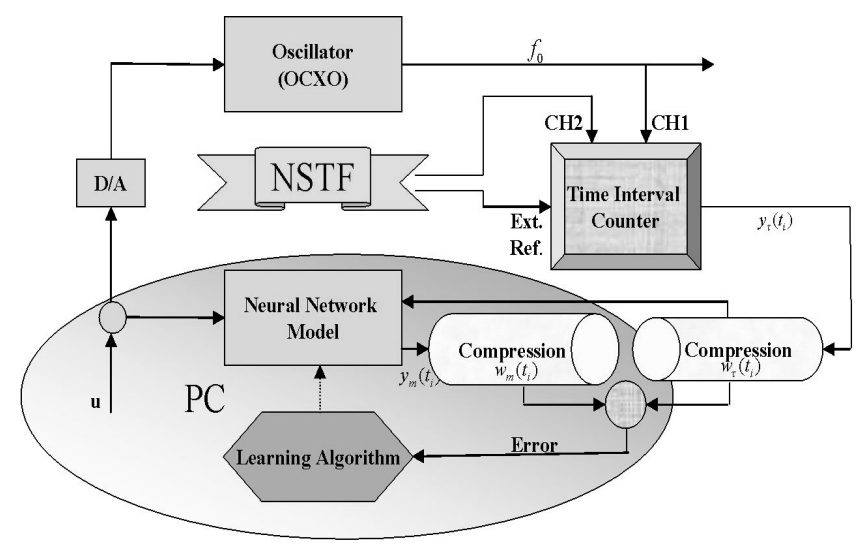

Figure 8 . The process of the MPC system identification

The first stage in modeling predictive control is to train a neural network to represent the forward dynamics of the OCXO. The prediction error between the compressed signal of the OCXO output phase error and the compressed signal of the neural network output is used as the neural network training signal. The process is represented in the following Figure 8. The neural network plant model uses previous inputs and previous plant outputs to predict future values of the plant output. The network can be trained offline in batch mode, using data collected from the operation of the plant.

The second stage is actual predictive control. The neural 
network model predicts the plant response over a specified time horizon. The predictions are used by a numerical optimization program to determine the control signal that minimizes the following performance criterion over the specified horizon.

$J=\sum_{k=N_{l}}^{N_{2}}\left(y_{m}(t+k)-y_{r}(t+k)\right)^{2}+\rho \sum_{k=1}^{N_{u}}\left(u^{\prime}(t+k-1)-u^{\prime}(t+k-2)\right)^{2}$

where $N_{1}, N_{2}$ and $N_{u}$ define the horizons over which the tracking error and the control increments are evaluated. The $u^{\prime}$ variable is the tentative control signal, $y_{r}$ is the desired response, and $y_{m}$ is the network model response. The $\rho$ value determines the contribution that the sum of the squares of the control increments makes to the performance index.

The following block diagram illustrates the model predictive control process. The controller consists of the neural network plant model and the optimization block. The optimization block determines the values of $u^{\prime}$ that minimize $J$, and then the optimal $u$ is input to the plant.

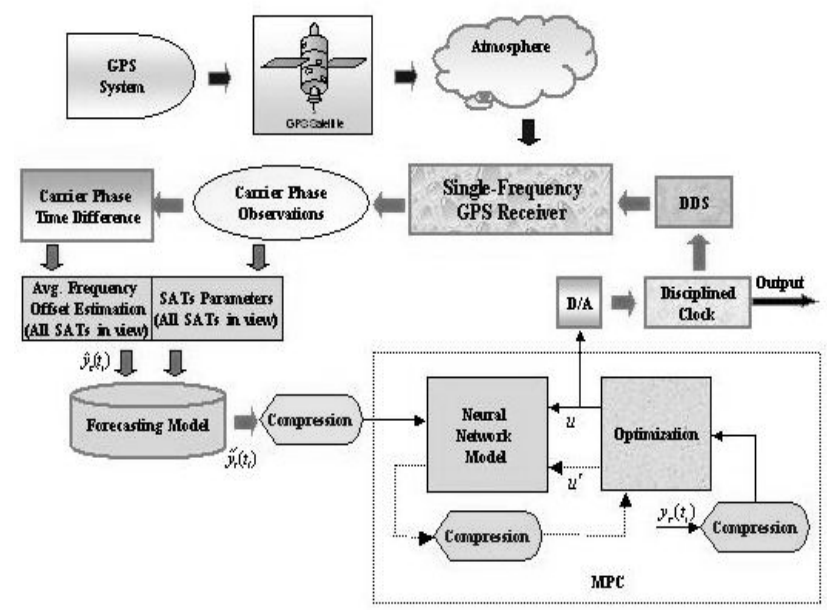

Figure 9. The model predictive control block diagram

\section{B. Initialization and Modeling}

On the other hand, the host (master) station also contains a high-performance frequency source with the national standards of time and frequency, the direct digital synthesizer (DDS), a modified G12 GPS receiver, and an industrial PC, all of which are the same as those owned by the user site. The carrier phase data and other GPS observations messages are then passed to the user site through a communication network, e.g. the PSTN or the Internet. The average frequency offset of the user clock with respect to the master clock can be estimated by performing the time difference (i.e. (2)) and then single difference (i.e. (3)) on the carrier phase observations. In the process, the biases and errors from the satellites and receivers can be significantly reduced. Equation (3) can be further expressed as follows:

$$
\Delta \delta \Phi_{A B}^{j}-\Delta \delta \rho_{A B}^{j}=-c \Delta \delta d T_{A B}+\Delta \delta \varepsilon_{A B}^{j}
$$

The term $\Delta \delta d T_{A B}\left(t_{i}\right)\left(=\delta d T_{A}\left(t_{i}\right)-\delta d T_{B}\left(t_{i}\right)\right.$, where $\left.t_{i}=t_{i-1}+\tau\right)$ can be obtained by averaging equation (21) for all-in-view GPS observations. Then, the associated estimate average frequency offset $\hat{y}_{r}^{\prime \prime}\left(t_{i}\right)$ is:

$$
\hat{y}_{r}^{\prime \prime}\left(t_{i}\right)=\frac{\Delta \delta d T_{A B}\left(t_{i}\right)}{\tau}-\frac{\Delta \delta \varepsilon_{A B}^{j}}{c \tau}
$$

To attain low-cost, highly-accurate training data on atmospheric variation for common users, we can first obtain the frequency stability and accuracy by performing equation (17) in real-time at both sites. Next, we acquire the reference destination of the atmosphere modeling by subtracting $\hat{y}_{r}^{\prime \prime}\left(t_{i}\right)$ from $\hat{y}_{r}^{\prime}\left(t_{i}\right)$ in the post-processing at the user site. Since the master station possesses the same degree of time and frequency as the GPS system time does, our model will not vary in accuracy according to user location. The model creation is adapted to the individual circumstances of the user site. The correction method of the syntonization system is thus more accurate and easier to use than the other models for a single-frequency receiver.

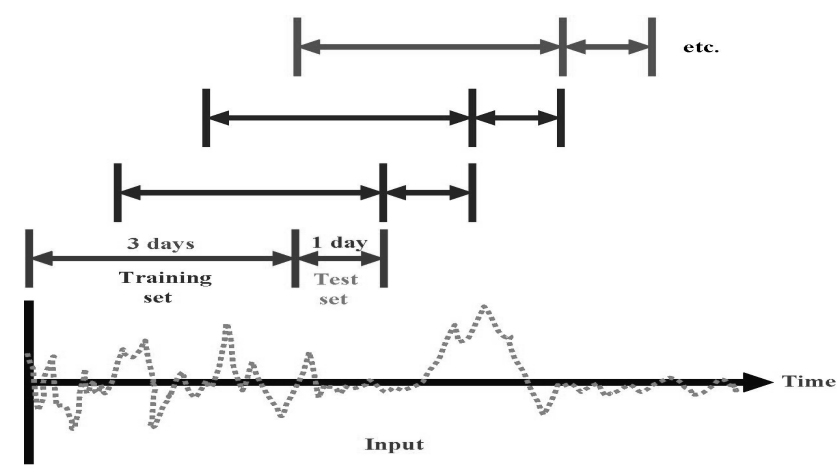

Figure 10. A depiction of the training and test sets used

The difficulty with our approach is the reduction in the already small quantity of training data. The size of the training set controls a noise vs. non-stationary trade-off [9], [10]. If the training set is too small, the noise makes it harder to estimate the appropriate mapping. If the training set is too large, the non-stationary data will lead to more data with statistics that are less relevant for the task at hand being used in the creation of the estimator. The entire training/test set window is moved forward 1 day and the process is repeated, as depicted in Figure 10. For the 
problem of noisy data, we use early stopping to control over-fitting.

\section{EXPERIMENTAL RESULTS}

The basic experimental structure of the tests is shown in Figure 9. The low-cost Ashtech ${ }^{\mathrm{TM}}$ G-12 single-frequency GPS receiver installed in our system was not designed for time and frequency applications. It had no interface ports for external clocks. In order to use the G-12 receivers to establish the system, we replaced the $20.460 \mathrm{MHZ}$ internal quartz oscillator of the receiver with the external frequency source and connected a G-12 receiver through a DDS manufactured by $\mathrm{NOVATECH}^{\mathrm{TM}}$, model DDs5m. An oven controlled crystal oscillator manufactured by Datum $^{\mathrm{TM}}$, model FTS 1130, was used as the disciplined clock. The software, including the model predictive controller, the real-time forecasting model, and the communication interface between the time interval counter (TIC) and a PC, which was used for data collection, were programmed in C++ language on Professional Windows 2000 and executed on a mobile computer manufactured by Acer. The data used for frequency accuracy and stability analysis were measured once every second with a TIC manufactured by SRS ${ }^{\mathrm{TM}}$, model SR620. This system included the DDS (Direct Digital Synthesizer), a D/A converter, a low-cost modified G12 GPS receiver, a forecasting model of atmosphere correction, and a notebook PC. In order to estimate the offsets of the steered clock with respect to the GPS system time, the user clock was connected to the modified GPS receiver. Hence, the original internal quartz oscillator in the receiver was replaced. The coordinates of the GPS antenna were predetermined by the IGS (International GPS Service). With the help of the frequency synthesizer, i.e. the DDS (Direct Digital Synthesizer) manufactured by NOVATECH, the signal of the external clock could be appropriately converted and supplied to the GPS receiver. The behavior of the clock then came into view from the GPS observations. The average frequency offset of the disciplined clock with respect to the GPS system time could be estimated by performing the time difference and real-time atmospheric corrections on the carrier phase observations with all satellites in view.

Finally, we examined the performance of the free running OCXO used in our system and a GPSDO manufactured by Trimble ThunderBolt ${ }^{T M}$ in order to compare the results with the performance of the controlled OCXO under atmospheric correction and the common view (CV) method. The frequency stability analysis is shown in Figure 11. The frequency stability of the OCXO without the atmosphere delay variation correction is significantly degraded over the averaging time between the $100 \mathrm{~s}$ to the $60,000 \mathrm{~s}$. Figure 12 shows the average-free phase difference between the free running OCXO clock and the primary (Cs.) clock. The accuracy of this OCXO is about $2.41 \times 10^{-9}$ for an averaging time of about one day. Figure 13 presents the average-free phase difference between the primary (Cs.) clock and the disciplined OCXO with atmospheric effects. The accuracy of the OCXO is about $5.43 \times 10^{-13}$ for an averaging time of about one day. Figure 14 presents the average-free phase difference between the primary clock and the controlled OCXO with atmospheric delay variation correction of the real-time forecasting model. The accuracy of the OCXO is about $3.12 \times 10^{-14}$ for an averaging time of about one day. The stability per second was about nine parts in $10^{12}$ in Figure 10. In addition, we found the frequency stability of the OCXO to be significantly improved over the averaging time between the100s to the $60,000 \mathrm{~s}$. With the above results, we demonstrated that our low-cost scheme, composed of a GPS carrier phase single-frequency receiver with the real-time atmospheric forecasting model, achieves a highly-accurate and reliable disciplined clock.

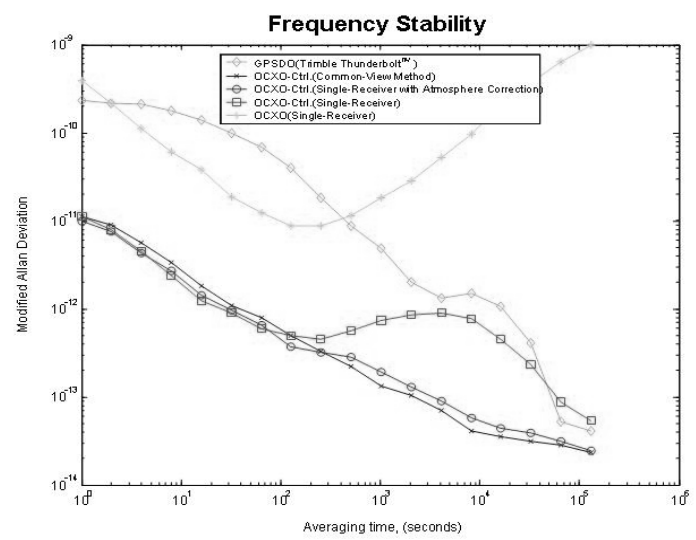

Figure 11. The frequency stability comparison of the free running OCXO, GPSDO Thunderbolt ${ }^{T M}$, OCXO-Ctrl without atm. delay correction, OCXO-Ctrl. with the atm. correction of the real-time forecasting model and the common-view method.

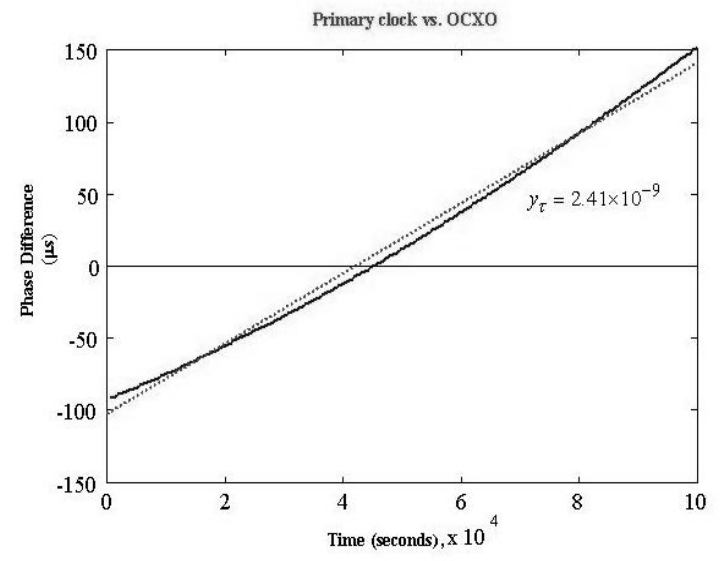

Figure 12. The phase difference between free running OCXO and primary clock with linear-fit line. 


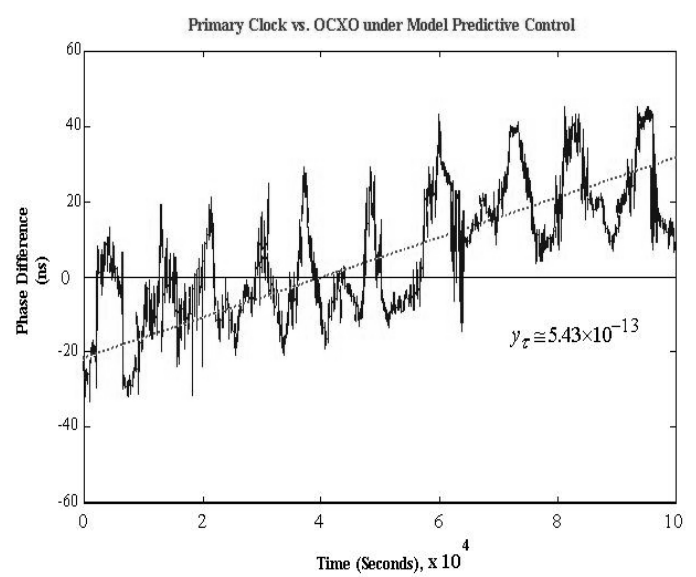

Figure 13. The phase difference between primary clock and OCXO-Ctrl. (MPC) under atmospheric effects with linear-fit line.

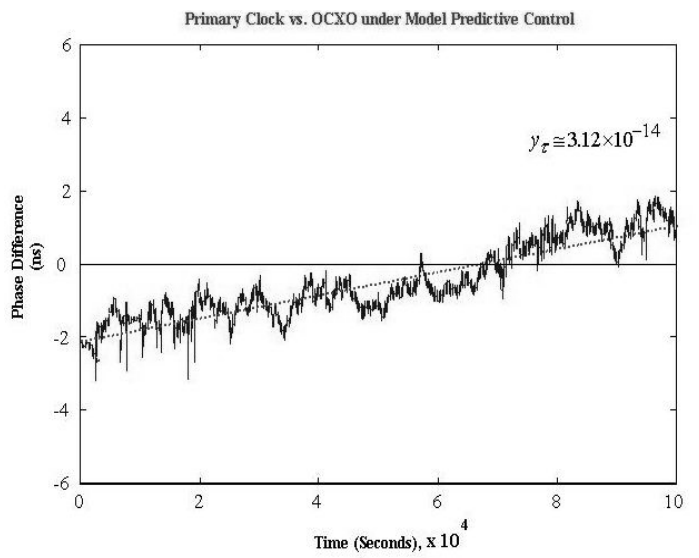

Figure 14. The phase difference between primary clock and OCXO-Ctrl. (MPC) under the real-time correction of forecasting atmospheric model with linear-fit line.

\section{CONCLUSIONS}

In this paper, a new low-cost, highly-accurate, and real-time GPS carrier phase disciplined system based on a single-frequency receiver and the atmospheric forecasting model is presented. The scheme can achieve traceability of frequency dissemination. In addition, we observed that the model predictive control of the neural network is robust and adaptive for our frequency syntonization by GPS carrier phase measurements. Three improvements and advantages in our methodology were given. First, we have developed a real-time forecasting model to correct atmospheric errors and filter time-series noise in real time. The model is more accurate than the existing ionosphere model and troposphere model for single GPS receivers. Furthermore, it is available easily and anticipative day-to-day, irrelevant to user locations. Second, the low-cost oscillator can be automatically steered to obtain very high frequency accuracy and stability in the short term as well as in the long term. Experimental results show that the stability per second was nine parts in $10^{12}$. Compared with the commercial GPS Disciplined Oscillators (GPSDOs), the short-term stability (1s) was improved by about a factor of ten. Moreover, the increased instability of the GPSDOs and other single receivers in the medium term (approximately from $100 \mathrm{~s}$ to $60000 \mathrm{~s}$ ) due to the atmosphere effect was also improved. Third, the frequency performance of the disciplined system, with the use of low-cost GPS engines, inexpensive clocks and less communication effects, was almost as good as that of the commercial atomic clock. Therefore, the disciplined frame of the clock is sound, reliable and cost-effective.

\section{ACKNOWLEDGEMENT}

We gratefully acknowledge the NSTF Lab., TL, Chunghwa Telecom. Co., Ltd., Taiwan, for experiment supporting of this paper.

\section{REFERENCES}

[1] K. Larson, and J. Levine, "Time-transfer using GPS carrier phase methods," Procccdings 29th Annual Precise Time and Time Interval (PTTI) Meeting, California, 1997.

[2] D. Wells, "Guide to GPS positioning," Canadian GPS Associates, 1996.

[3] N.K. Bose and P. Liang,"Neural network fundamentals with graphs, algorithm, and applications," McGraw-Hill Electrical and Computer Engineering Series, McGraw-Hill Inc. 1996.

[4] G. Zhang et al, "Wavelet transform for filtering financial data streams," Journal of Computational Intelligence in Finance, Vol. 7, NO. 3, May/Jun., 1999.

[5] C. Lee Giles, Steve Lawrence, A.C. Tsoi, "Noisy time series prediction using a recurrent neural network and grammatical inference," Machine Learning, Vol. 44, pp161-183, Jul./Aug., 2001.

[6] J.L. Elman, "Distributed representations, simple recurrent networks and grammatical structure," Machine Learning, 7(2/3): 195-226, 1991

[7] G.U. Yule, "On a method of investigating periodicities in disturbed series with special reference to Wolfer's sunspot numbers," Philosophical Transactions Royal Society London Series A, 226:267-298, 1927

[8] Soloway, D. and P.J. Haley, "Neural Generalized Predictive Control," Proceedings of the IEEE International symposium on Intelligent Control, pp. 277-281, 1996.

[9] J. E. Moody, "Economic forecasting: Challenges and neural network solutions," in Proceedings of the international symposium on Artificial neural network, Hsinchu, Taiwan, 1995.

[10] C.W.J. Granger and P. Newbold., "Forecasting economic time series," Academic Press, second edition, 1986. 